PROCEEDINGS OF THE AMERICAN MATHEMATICAL SOCIETY

Volume 124, Number 11, November 1996

\title{
JOINT CONTINUITY OF SEPARATELY CONTINUOUS MAPPINGS ON TOPOLOGICAL GROUPS
}

\author{
H. R. EBRAHIMI-VISHKI
}

(Communicated by Franklin D. Tall)

\begin{abstract}
The main theorem of this paper is somewhat stronger than the following statement: Let $G$ be a Baire semitopological group, let $H$ be a first countable one and let $N$ be a first countable topological group; then each separately continuous bi-homomorphism from $G \times H$ into $N$ is jointly continuous. This theorem has some consequences on joint continuity of separately continuous multiplication of rings and scalar multiplication of modules.
\end{abstract}

\section{INTRODUCTION}

There are many papers which deal with the classical problem of determination of points of continuity of separately continuous mappings. The general problem is to find conditions on topological spaces $X, Y$ and $Z$ such that each separately continuous mapping from $X \times Y$ into $Z$ is jointly continuous on some substantial subset of $X \times Y$. Some authors, in their treatment of the above problem, considered some algebraic conditions on the spaces and the mappings involved. In this paper our aim is to provide an answer for a special case of the above general problem.

\section{Preliminaries}

2.1. Definition. Let $X, Y$ be two topological spaces and let $Z$ be a uniform space with a uniformity $\Omega$. We call the triple $(X, Y, Z)$ a Namioka-Troallic triple if there exists a nonempty subset $C$ of $Y$ such that if $U \in \Omega$, then each separately continuous mapping $f: X \times Y \rightarrow Z$ is $U$-jointly continuous on $A_{U} \times C$ for some dense $G_{\delta}$ subset $A_{U}$ of $X$, i.e. there is a neighbourhood $U_{1} \times U_{2}$ of $(x, y)$ such that

$$
f\left(U_{1} \times U_{2}\right) \subset U[f(x, y)]=\{z \in Z:(f(x, y), z) \in U\} \quad\left((x, y) \in A_{U} \times C\right) .
$$

If $X$ and $Y$ are two spaces such that $X$ is Baire and each separately continuous mapping from $X \times Y$ into any pseudo-metric space is jointly continuous on $A \times C$ for some dense $G_{\delta}$ subset $A$ of $X$ and for some subset $C$ of $Y$, then it is trivial that $(X, Y, Z)$ is a Namioka-Troallic triple for each uniform space $Z$, since each uniform space is uniformly isomorphic to a subspace of the product of pseudo-metric spaces. Hence we have the following two propositios (see for example [1], [4] or [5] for 2.2 and [6] for 2.3).

Received by the editors March 21, 1994.

1991 Mathematics Subject Classification. Primary 54C05, 22A10.

Key words and phrases. Separate and joint continuity, Baire space, $\sigma$-well- $\beta$-defavorable space, topological group. 
2.2. Proposition. If $X$ is a $\sigma$-well- $\beta$-defavorable space (in particular, if $X$ is a complete pseudo-metric or a locally compact space) and $Y$ is a compact Hausdorff space, then for every uniform space $Z$ the triple $(X, Y, Z)$ is a Namioka-Troallic triple.

2.3. Proposition. If $X$ is a Baire space and $Y$ is at least first countable (i.e. $Y$ has a countable base of neighbourhoods at some point), then for every uniform space $Z$ the triple $(X, Y, Z)$ is a Namioka-Troallic triple.

Remark. (i) If $(X, Y, Z)$ is a Namioka-Troallic triple, then in general it is not true that each separately continuous mapping from $X \times Y$ into $Z$ is jointly continuous at some points of $X \times Y$ (see, for example, Hoffman-Jørgensen's example in [1]). But, if $(X, Y, Z)$ is a Namioka-Troallic triple such that $X$ is Baire and $Z$ is pseudometric, then it is trivial that each separately continuous mapping from $X \times Y$ into $Z$ is jointly continuous on some subset $A \times C$ of $X \times Y$ where $A$ is a dense $G_{\delta}$ subset of $X$.

(ii) For constructing a non-Namioka-Troallic triple, see [2]. Indeed, if $X$ is the set $\mathbf{Q}$ of rational numbers, $Y$ is the unit interval, and $Z$ is the space of all continuous functions from $\mathbf{Q}^{2}$ into $[-1,1]$ equipped with the pointwise topology, then $(X, Y, Z)$ is not a Namioka-Troallic triple.

Here we pose a question: Suppose $X$ is a completely regular space such that $(X, Y, Z)$ is a Namioka-Troallic triple for every compact Hausdorff space $Y$ and every pseudo-metric space $Z$. Is it true that $X$ is Baire? (See also Theorem 3 of [5].)

\section{THE MAIN RESULTS}

Suppose $G, H$, and $N$ are groups. A mapping $B: G \times H \mapsto N$ is said to be a bi-homomorphism if for each fixed $\left(g_{0}, h_{0}\right) \in G \times H$, the mappings $h \mapsto B\left(g_{0}, h\right)$ : $H \mapsto N$ and $g \mapsto B\left(g, h_{0}\right): G \mapsto N$ are homomorphisms of groups.

In the following lemma $\Omega$ is assumed to be the left uniformity on the topological group $N$. That is, $\Omega$ is the set of all $L_{U}=\left\{(x, y) \in N \times N, x^{-1} y \in U\right\}$, where $U$ is a neighbourhood of the identity of $N$.

3.1. Lemma. Let $G, H$ be two semitopological groups (i.e. two semitopological semigroups which algebraically are groups), let $N$ be a topological group, and let $B$ : $G \times H \rightarrow N$ be a separately continuous bi-homomorphism. Then

(i) $B$ is jointly continuous on $G \times H$ if and only if $B$ is jointly continuous at least in one point of $G \times H$.

(ii) If $C$ is a subset of $H$ such that for each $L_{U} \in \Omega, B$ is $L_{U}$-jointly continuous on $A_{U} \times C$ for some nonempty subset $A_{U}$ of $G$ and if $A=\bigcap A_{U}$ (where the intersection is taken over all neighbourhoods $U$ of the identity of $N$ ) is nonempty, then $B$ is jointly continuous on $A \times C$ and so on $G \times H$.

Proof. The proof of (i) is trivial. For (ii) suppose $\left\{\left(g_{\alpha}, h_{\alpha}\right)\right\}$ is a net in $G \times H$ which converges to $\left(g_{0}, h_{0}\right) \in A \times C$. For each $\alpha$, we have

$$
\begin{aligned}
& B\left(g_{\alpha}, h_{\alpha}\right) B\left(g_{0}, h_{0}\right)^{-1} \\
& \quad=\left[B\left(g_{0}, h_{\alpha}\right)^{-1} B\left(g_{0}, h_{0}\right)\right]\left[B\left(g_{0}, h_{0}\right)^{-1} B\left(g_{0} g_{\alpha} g_{0}^{-1}, h_{\alpha}\right)\right] B\left(g_{0}, h_{\alpha} h_{0}^{-1}\right) .
\end{aligned}
$$


Let $U, V$ be two neighbourhoods of the identity of $N$ such that $U$ is symmetric (i.e. $U=U^{-1}$ ) and $U^{3} \subset V$. For sufficiently large $\alpha$,

$$
\left.B\left(g_{0}, h_{\alpha} h_{0}^{-1}\right) \in U \quad \text { and } B\left(g_{0}, h_{\alpha}\right)^{-1} B\left(g_{0}, h_{0}\right)\right] \in U
$$

since $B$ is separately continuous. Also $\left[B\left(g_{0}, h_{0}\right)^{-1} B\left(g_{0} g_{\alpha} g_{0}^{-1}, h_{\alpha}\right)\right] \in U$, since $B$ is $L_{U}$-jointly continuous on $A_{U} \times C$. So $B\left(g_{\alpha}, h_{\alpha}\right) B\left(g_{0}, h_{0}\right)^{-1} \in U^{3} \subset V$. That is, $B$ is jointly continuous on $A \times C$.

3.2. Theorem. Let $G, H$ be two semitopological groups, let $N$ be a topological group, and let $B: G \times H \rightarrow N$ be a separately continuous bi-homomorphism. If $(G, H, N)$ is a Namioka-Troallic triple, then $B$ is jointly continuous if either:

(i) the intersection of any number of dense $G_{\delta}$-subsets of $G$ is nonempty; or

(ii) $G$ is Baire and $N$ is first countable.

Proof. Let $\tau$ be the set of all neighbourhoods of the identity of $N$. There is a subset $C$ of $H$ such that for each $U \in \tau, B$ is $L_{U}$-jointly continuous on $A_{U} \times C$ for some dense $G_{\delta}$ subset $A_{U}$ of $G$. If (i) holds, then $\bigcap_{U \in \tau} A_{U} \neq \varnothing$. If (ii) holds, then we may assume that $\tau$ is countable, hence $\bigcap_{U \in \tau} A_{U} \neq \varnothing$. Thus in either case $B$ is jointly continuous on $\left(\bigcap_{U \in \tau} A_{U}\right) \times C$ (Lemma 3.1$)$, so it is jointly continuous.

Remark. Theorem 3.2 is clearly a generalization of the following statement which appears as Theorem 2 of [3]. (Let $G, H$, and $N$ be abelien complete metrizable groups. Let $B: G \times H \mapsto N$ be bi-additive and separately continuous. Then $B$ is jointly continuous.)

3.3. Example. Let $H$ be an infinite-dimensional Hilbert space with its weak topology; then the inner product is a separately but not jointly continuous bi-homomorphism from $H \times H$ (consider the additive group of $H$ ) into the topological group of complex numbers.

Proposition 2.3 and Theorem 3.2(ii) give us the following corollaries.

3.4. Corollary. Each Baire first countable semitopological ring is a topological ring.

3.5. Corollary. If $R$ is a Baire ring such that its addition is separately continuous, then each first countable semitopological $R$-module is a topological $R$-module.

Conjecture. We conjecture that the result of Theorem 3.2(ii) remains valid without first countability of $N$.

\section{ACKNOWLEDGMENTS}

The author wishes to express his gratitude to Professors M. A. Pourabdollah and A. T. M. Lau for their valuable comments. Thanks are also due to the referee for his helpful comments.

\section{REFERENCES}

1. J. P. R. Christensen, Joint continuity of separately continuous functions, Proc. Amer. Math. Soc. 82 (1981), 455-461. MR 82h:54012

2. - Remarks on Namioka spaces and R. E. Johnson's theorem on the norm separability of the range of certain mappings, Math. Scand. 52 (1983), 112-116. MR 85c:46003

3. J. P. R. Christensen and P. Fischer, Joint continuity of measurable biadditive mappings, Proc. Amer. Math. Soc. 103 (1988), 1125-1128. MR 89d:43006 
4. I. Namioka, Separate continuity and joint continuity, Pacific J. Math. 51 (1974), 515-531. MR 51:6693

5. J. S. Raymond, Jeux toplogiques et spaces de Namioka, Proc. Amer. Math. Soc. 87 (1983), 499-504. MR 83m:54060

6. J. Calbrix et J. P. Troallic, Applications séparément continues, C. R. Acad. Sci. Paris, Sér. A 288 (1979), 647-648. MR 80c:54009

Department of Mathematics, Mashhad University, P. O. Box 1159, Mashhad 91775, IRAN

E-mail address: vishki@science2.um.ac.ir 